\title{
ALGEBRA VERSUS ANALYSIS IN THE THEORY OF FLEXIBLE POLYHEDRA
}

\author{
VICTOR ALEXANDROV
}

\begin{abstract}
Two basic theorems of the theory of flexible polyhedra were proven by completely different methods: R. Alexander used analysis, namely, the Stokes theorem, to prove that the total mean curvature remains constant during the flex, while I.Kh. Sabitov used algebra, namely, the theory of resultants, to prove that the oriented volume remains constant during the flex. We show that none of these methods can be used to prove the both theorems. As a by-product, we prove that the total mean curvature of any polyhedron in the Euclidean 3-space is not an algebraic function of its edge lengths.
\end{abstract}

A polyhedron (more precisely, a polyhedral surface) is said to be flexible if its spatial shape can be changed continuously due to changes of its dihedral angles only, i. e., if every face remains congruent to itself during the flex. In other words, a polyhedron $P_{0}$ is flexible if it is included in a continuous family $\left\{P_{t}\right\}, 0 \leqslant t \leqslant 1$, of polyhedra $P_{t}$ such that, for every $t$, the corresponding faces of $P_{0}$ and $P_{t}$ are congruent while the polyhedra $P_{0}$ and $P_{t}$ are not congruent. The family $\left\{P_{t}\right\}$, $0 \leqslant t \leqslant 1$, is called the flex of $P_{0}$. Self-intersections are possible both for $P_{0}$ and $P_{t}$ provided the converse is not formulated explicitly. Without loss of generality we assume that the faces of the polyhedra are triangular.

Flexible self-intersection free sphere-homeomorphic polyhedra in Euclidean 3space were constructed by R. Connelly thirty years ago [4, 6]. Since that time, various non-trivial properties of flexible polyhedra were discovered in the Euclidean 3 -space [10] and 4-space [12] (for results in the hyperbolic 3-space, see [13]). Let us formulate two of them in a form suitable for our purposes.

Let $P$ be a closed oriented polyhedron in $\mathbb{R}^{3}$, let $E$ be the set of its edges, let $|\ell|$ be the length of the edge $\ell$, and let $\alpha(\ell)$ be the dihedral angle of $P$ at the edge $\ell$ measured from inside of $P$. The sum

$$
M(P)=\frac{1}{2} \sum_{\ell \in E}|\ell|(\pi-\alpha(\ell))
$$

is called the total mean curvature of $P$.

Theorem 1. Let $P_{0}$ be a flexible polyhedron in $\mathbb{R}^{3}$ and let $\left\{P_{t}\right\}, 0 \leqslant t \leqslant 1$, be its flex. The total mean curvature $M\left(P_{t}\right)$ is independent of $t$.

Theorem 1 was proved by R. Alexander 2 for all Euclidean $n$-spaces, $n \geqslant 3$, though no example of a flexible polyhedron is known for $n \geqslant 5$.

Date: July 6, 2010.

1991 Mathematics Subject Classification. Primary 52C25; Secondary 51M20.

Key words and phrases. Flexible polyhedron, volume, infinitesimal bending, total mean curvature, algebraic function.

The author was supported by the Federal Program 'Research and educational resourses of innovative Russia in 2009-2013' (contract 02.740.11.0457) and the Russian Foundation for Basic Research (grant 10-01-91000-ANF). 
Let $P_{0}$ be a flexible polyhedron and let $\left\{P_{t}\right\}, 0 \leqslant t \leqslant 1$, be its flex. Let $\boldsymbol{r}_{t}$ be the point of the polyhedron $P_{t}$ which corresponds to the point $\boldsymbol{r}_{0} \in P_{0}$. It follows from the definition of a flexible polyhedron that, for any curve $\gamma_{0} \subset P_{0}$, the length of the curve $\gamma_{t}=\left\{\boldsymbol{r}_{t} \mid \boldsymbol{r}_{0} \in \gamma_{0}\right\} \subset P_{t}$ is independent of $t$. The reader can easily verify the well-known fact that, for any curve $\gamma_{0} \subset P_{0}$, the length of the curve $\widetilde{\gamma}_{t}=\left\{\boldsymbol{r}_{0}+t \boldsymbol{v} \mid \boldsymbol{r}_{0} \in \gamma_{0}\right\}$ is stationary at $t=0$, where $\boldsymbol{v}=\left.\frac{d}{d t}\right|_{t=0} \boldsymbol{r}_{t}$ is the velocity vector of the point $\boldsymbol{r}_{t}$ at $t=0$. Obviously, the vector field $\boldsymbol{v}$ is linear on every face of $P_{0}$. This leads to the following well-known definition: a vector field $\boldsymbol{w}$ on a polyhedron $P$, which is linear on every face of $P$, is said to be its infinitesimal flex if, for any curve $\boldsymbol{\gamma} \subset P$, the length of the curve $\widetilde{\boldsymbol{\gamma}}(t)=\{\boldsymbol{r}+t \boldsymbol{w} \mid \boldsymbol{r} \in \boldsymbol{\gamma}\}$ is stationary at $t=0$, see [7] for more detail. Of course, the velocity vector field of a flexible polyhedron is its infinitesimal flex (but the converse is not necessarily true).

In 2] Theorem 1 was obtained as an obvious corollary of the following Theorem 2 proved for $\mathbb{R}^{n}, n \geqslant 3$.

Theorem 2. Let $P$ be a closed oriented polyhedron in $\mathbb{R}^{3}$, let $\boldsymbol{w}$ be its infinitesimal flex, and let $P(t)=\{\boldsymbol{r}+t \boldsymbol{w} \mid \boldsymbol{r} \in P\}$. Then $\left.\frac{d}{d t}\right|_{t=0} M(P(t))=0$.

In 2] Theorem 2 was proved with the help of the Stokes theorem. Later several authors observed (see, e.g., [3, [11]) that Theorem 2 follows immediately from the Schläfli differential formula, which, in turn, is based on the Stokes theorem. In any case, all known proofs of Theorems 1 and 2 belong to Analysis.

In 8 I.Kh. Sabitov proved another highly non-trivial property of the flexible polyhedra that may be formulated as follows and whose many-dimensional analog is not known yet.

Theorem 3. If $\left\{P_{t}\right\}$ is a flex of an orientable polyhedron in $\mathbb{R}^{3}$, then the oriented volume of $P_{t}$ is constant in $t$.

In [8, 9], and later in [5] Theorem 3 was obtained as an obvious corollary of the following Theorem 4 valid since every continuous mapping, whose image is a finite set, is constant.

Theorem 4. For the set $\mathcal{P}_{K}$ of all (not necessarily flexible) closed polyhedra in $\mathbb{R}^{3}$ with triangular faces and with a prescribed combinatorial structure $K$ there exists a universal polynomial $\mathfrak{p}_{K}$ of a single variable whose coefficients are universal polynomials in the edge lengths of a polyhedron $P \in \mathcal{P}_{K}$ and such that the oriented the volume of any $P \in \mathcal{P}_{K}$ is a root of $\mathfrak{p}_{K}$.

In [8] and [9] Theorem 4 was proved with the help of the theory of resultants, while in 5] it was proved with the help of the theory of places. In any case, all known proofs of Theorems 3 and 4 belong to Algebra.

Now recall that the derivative of the volume of a deformable domain in $\mathbb{R}^{3}$ equals one third of the flux across the boundary of the velocity vector of a point of the boundary of the domain, i. e., equals one third of the integral of the normal component of the velocity over the boundary. Hence, an 'infinitesimal version' of Theorem 3 should read that, for every orientable polyhedron $P$, the flux $\int_{P}(\boldsymbol{w}, \boldsymbol{n}) d P$ of any infinitesimal flex $\boldsymbol{w}$ equals zero. Theorem 5 shows that this is not the case and, thus, that Theorem 3 can not be proved by means of Analysis like Theorem 1 .

Theorem 5. There is a closed oriented polyhedron $P$ in $\mathbb{R}^{3}$ with the following properties:

(i) the flux across $P$ of some infinitesimal flex $\boldsymbol{w}$ of $P$ is non-zero;

(ii) $P$ contains no vertex $V$ whose star lies in a plane; 


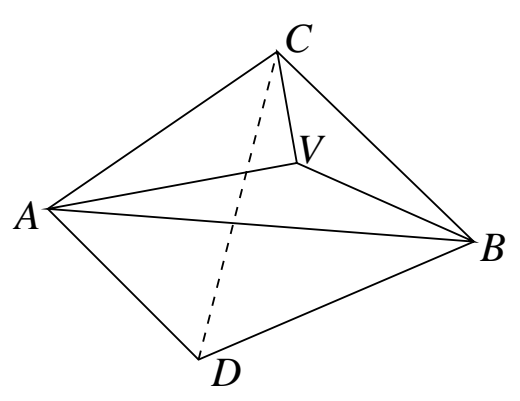

Figure 1. Polyhedron $T_{1}$

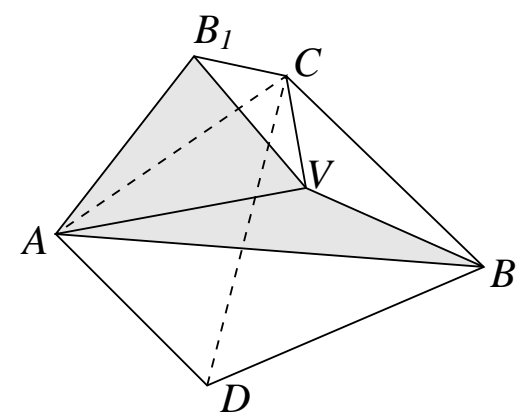

Figure 2. Polyhedron $T_{2}$

(iii) $P$ contains no vertex $V$ such that some three edges of $P$ incident to $V$ lie in a plane.

Proof. Let $T_{0}=A B C D$ be an arbitrary tetrahedron in $\mathbb{R}^{3}$. Let a polyhedron $T_{1}$ be obtained from $T_{0}$ by triangulation of the face $A B C$ that uses one additional vertex $V$ (see Fig. 1). Let a vector field $\boldsymbol{w}_{1}$ be linear on each face of $T_{1}$, be equal zero at the vertices $A, B, C$, and $D$, and be equal a non-zero vector perpendicular to the face $A B C$ at the vertex $V$. It is easy to check that $\boldsymbol{w}_{1}$ is an infinitesimal flex of $T_{1}$ and its flux across $T_{1}$ is non-zero (because the scalar product $\left(\boldsymbol{w}_{1}, \boldsymbol{n}\right)$ is either everywhere non-negative or everywhere non-positive; here $\boldsymbol{n}$ stands for the unit normal vector field on $T_{1}$ ). Hence, $T_{1}$ satisfies the condition (i), though it does not satisfy the conditions (ii) and (iii).

Let $B_{1}$ be an arbitrary point in $\mathbb{R}^{3}$ which does not lie in the plane $A B C$. Replace the face $A C V$ of the polyhedron $T_{1}$ with the lateral surface of the triangular pyramid $A C V B_{1}$ with the base $A C V$. Denote the resulting polyhedron by $T_{2}$ (see Fig. 2). It is easy to check that the infinitesimal flex $\boldsymbol{w}_{1}$ of $T_{1}$ can be extended in a unique way to an infinitesimal flex of $T_{2}$. Denote it by $\boldsymbol{w}_{2}$ and observe that the flux of $\boldsymbol{w}_{2}$ across $T_{2}$ equals the flux of $\boldsymbol{w}_{1}$ across $T_{1}$ (because the flux of $\boldsymbol{w}_{2}$ across the triangular pyramid $A C V B_{1}$ equals zero). Hence, $T_{2}$ satisfies the conditions (i) and (ii), though it does not satisfy the condition (iii).

Recall the construction of the Bricard flexible octahedron of type 1. Consider a disk-homeomorphic piece-wise linear surface $S$ in $\mathbb{R}^{3}$ composed of four triangles $A B V, B A_{1} V, A_{1} B_{1} V$, and $B_{1} A V$ such that $|A B|=\left|A_{1} B_{1}\right|$ and $\left|B_{1} A\right|=\left|B A_{1}\right|$ (see Fig. 3). It is known [6] that such a spatial quadrilateral $A B A_{1} B_{1}$ is symmetric with respect to the line $L$ passing through the middle points of its diagonals $A A_{1}$ and $B B_{1}$. Glue together $S$ and its symmetric image with respect to $L$ (see Fig. 4 ). The resulting polyhedral surface with self-intersections is flexible (because $S$ is flexible) and is combinatorially equivalent to the surface of the regular octahedron. This is known as the Bricard octahedron of type 1.

Construct the Bricard octahedron of type 1 such that its triangles $A B V$ and $A V B_{1}$ are congruent with the corresponding triangles $A B V$ and $A V B_{1}$ of the polyhedron $T_{2}$. Remove those triangles from $T_{2}$ and from the Bricard octahedron and glue together the remaining parts of those polyhedra. Observe that the resulting polyhedron $P$ satisfies the conditions (i)-(iii). In fact, the infinitesimal flex $\boldsymbol{w}_{2}$ of $T_{2}$ can be extended in a unique way to an infinitesimal flex of $P$. Denote it by $\boldsymbol{w}$ and observe that the flux of $\boldsymbol{w}$ across $P$ equals the flux of $\boldsymbol{w}_{2}$ across $T_{2}$ (because 


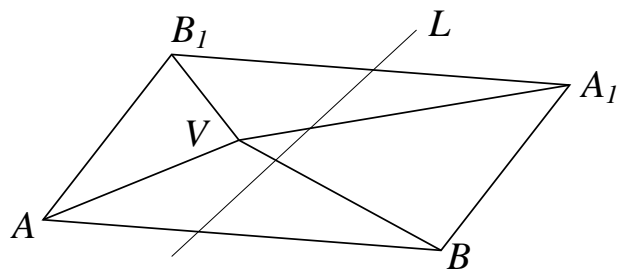

FiguRe 3. Disk $S$

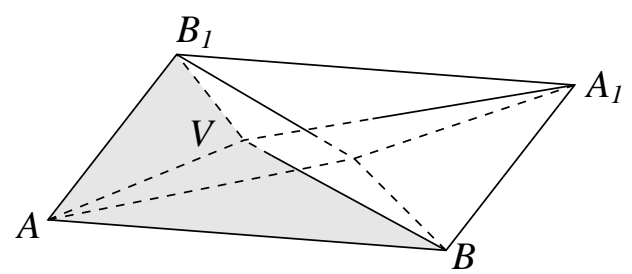

Figure 4. Bricard octahedron

the restriction of the flux $\boldsymbol{w}$ on the Bricard octahedron is generated by some its flex and, thus equals zero, since, e. g., the oriented volume of the Bricard octahedron is known to be constant for every flex [5], 7]-[10]).

Observe that an 'algebraic version' of Theorem 2 should read that the total mean curvature of any oriented polyhedron is a root of some universal polynomial $\mathfrak{p}$ in a single variable whose coefficients are universal polynomials in the edge lengths of the polyhedron. This statement would, obviously, imply Theorem 1. But this statement also implies that the total mean curvature of a polyhedron is an algebraic function of its edge lengths. Theorem 6 shows that this is not the case and, thus, that Theorem 1 can not be proved by means of Algebra like Theorem 3 .

Theorem 6. The total mean curvature of any closed oriented polyhedron in $\mathbb{R}^{3}$ is not an algebraic function of its edge lengths.

Proof. For every $K$, the class of all polyhedra of combinatorial type $K$ contains a family $\Delta_{K}$ of polyhedra depending on a single independent variable $l>0$ which may be described as follows (see Fig. 5): there are two triangles $A B C$ and $A C D$ in $\mathbb{R}^{3}$ such that

$(\alpha)|B C|=|C D|=|B D|=1$ and $|A B|=|A C|=|A D|=l$;

$(\beta)$ every $P \in \Delta_{K}$ has the triangles $A B C$ and $A C D$ as their faces;

$(\gamma)$ every vertex of $P \in \Delta_{K}$ either coincides with $A, B, C$, or $D$ or is an interior point of the segment $B D$ or is an interior point of the triangle $B C D$;

$(\delta)$ the set of the interior points of every edge of $P \in \Delta_{K}$ either coincides with $A B, A C$, or $A D$ or lies in the open segment $B D$ or lies in one of the open triangles $A B D$ or $B C D$.

A direct calculation shows that, for every $P \in \Delta_{K}$, the total mean curvature $M(P)$ of $P$ equals

$$
\begin{aligned}
M(P) & =\frac{3}{2}(\pi-\varphi(l))+\frac{3}{2} l(\pi-\psi(l)) \\
& =\frac{3}{2}\left(\pi-\arccos \frac{1}{2 \sqrt{3} \sqrt{4 l^{2}-1}}\right)+\frac{3}{2} l\left(\pi-\arccos \frac{2 l^{2}-1}{4 l^{2}-1}\right),
\end{aligned}
$$

where $\varphi(l)$ is the inner dihedral angle of $P$ at the edge $B C$ and $\psi(l)$ is the inner dihedral angle of $P$ at the edge $A C$. Consider the right-hand side of the last formula as a function of complex variable $l$. Obviously, this function has a non-algebraic singularity (known also as a logarithmic branch point) over $l=0$ (as well as over $l= \pm 1 / \sqrt{3}$ and $l= \pm i \sqrt{48 / 13})$.

Recall that a function $w=f(z)$ of a single complex variable $z$ is called algebraic, if there is a polynomial $\mathfrak{p}(w, z)$ in two variables which does not vanish identically and such that $\mathfrak{p}(f(z), z) \equiv 0$. It is known that an analytic function of a single 


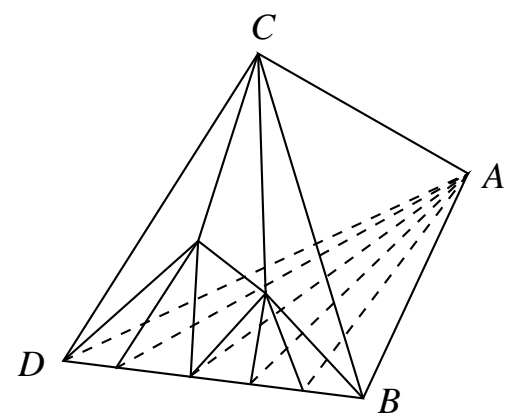

Figure 5. Polyhedron of the class $\Delta_{K}$

complex variable is an algebraic function if and only if it has a finite number of branches and at most algebraic singularities [1, p. 306]. Hence, the total mean curvature $M(P)$ is not an algebraic function of the variable $l$ for the polynomials $P$ of the class $\Delta_{K}$. Thus, $M(P)$ is not an algebraic function of the edge lengths of the polyhedron $P$.

\section{REFERENCES}

[1] L. Ahlfors, Complex analysis, 3rd ed. McGraw-Hill, 1979.

[2] R. Alexander, Lipschitzian mappings and total mean curvature of polyhedral surfaces, I, Trans. Amer. Math. Soc. 288 (1985), 661-678.

[3] F. Almgren and I. Rivin, The mean curvature integral is invariant under bending, in: The Epstein Birthday Schrift, University of Warwick, 1998, pp. 1-21.

[4] R. Connelly, Conjectures and open questions in rigidity, in: Proc. Int. Congr. Math., Helsinki 1978, Vol. 1, 1980, pp. 407-414.

[5] R. Connelly, I. Sabitov, and A. Walz, The bellows conjecture, Beitr. Algebra Geom. 38 (1997), $1-10$.

[6] N. H. Kuiper, Sphères polyedriques flexibles dans $E^{3}$, d'après Robert Connelly, in: Seminaire Bourbaki, Vol. 1977/78, Expose No.514, Lect. Notes Math. 710, 1979, pp. 147-168.

[7] I. Kh. Sabitov, Local theory on bendings of surfaces, in: Geometry III. Theory of surfaces. Encycl. Math. Sci., 48, 1992, pp. 179-250.

[8] I. Kh. Sabitov, The volume of a polyhedron as a function of its metric (in Russian), Fundam. Prikl. Mat. 2 (1996), 1235-1246.

[9] I. Kh. Sabitov, The volume as a metric invariant of polyhedra, Discrete Comput. Geom. 20 (1998), 405-425.

[10] J.-M. Schlenker, La conjecture des soufflets (d'après I. Sabitov), in: Seminaire Bourbaki, Vol. 2002/03. Société Math. de France, Paris. Astérisque 294, 77-95, Exp. No. 912 (2004).

[11] R. Souam, The Schläfli formula for polyhedra and piecewise smooth hypersurfaces, Differ. Geom. Appl. 20 (2004), 31-45.

[12] H. Stachel, Flexible cross-polytopes in the Euclidean 4-space, J. Geom. Graph. 4 (2000), 159-167.

[13] H. Stachel, Flexible octahedra in the hyperbolic space, in: A. Prékopa et al. Non-Euclidean geometries. János Bolyai memorial volume, Springer, New York, 2006, pp. 209-225.

Sobolev Institute of Mathematics, Koptyug ave., 4, Novosibirsk, 630090, Russia and Department of Physics, Novosibirsk State University, Pirogov str., 2, Novosibirsk, 630090, Russia

E-mail address: alex@math.nsc.ru 